\title{
Neuropsychiatric Systemic Lupus Erythematosus Complicated with Acute Pericarditis in Patient with Hyperprolactinaemia: A Case Report
}

\author{
Caterina Naclerio ${ }^{1}$, Giovanna Cuomo ${ }^{2}$, Gabriele Valentini ${ }^{2}$ \\ ${ }^{1}$ Rheumatology Unit “M. Scarlato” Hospital, Scafati, Italy; ${ }^{2}$ Chief of Rheumatology Unit, Second University of Naples, Naples, \\ Italy. \\ Email: caterinanaclerio@libero.it
}

Received December $6^{\text {th }}$, 2012; revised January $7^{\text {th }}$, 2013; accepted January $19^{\text {th }}, 2013$

\begin{abstract}
Neuropsychiatric systemic lupus erythematosus (NP-SLE) is one of the major cause of morbidity in systemic lupus erythematosus patients and its treatment depends on identification of pathogenic mechanisms. We describe the rare case of neuropsychiatric systemic lupus erythematosus (NP-SLE) complicated by pericardial effusion combined to low C4 level persisting and hyperprolactinaemia. A cyclophosphamide therapy showed a good response in a 21-year old woman with disturbances in thought processes and an acute confusional state with sierositis. This paper confirms that a cyclophosphamide therapy contributes to control a disease activity by a mechanism of prolactin level reduction. Other studies occur to evaluate this hypothesis.
\end{abstract}

Keywords: Systemic Lupus Erythematosus; Neuropsychiatric Systemic Lupus Erythematosus; Psychosis; Pericarditis; C4 Level; Prolactin

\section{Introduction}

Neuropsychiatric systemic lupus erythematosus (NP-SLE) refers to a constellation of neurologic, psychiatric and behavioral abnormalities that occur in patients with SLE [1]. Clinical abnormalities are reported in $40 \%-75 \%$ of patients during the course of the disease which are related to thrombotic and non-thrombotic mechanisms. Severe diffuse CNS manifestations, such as acute confusional state, generalised seizures, mood disorders and psychosis are associated to a variety of immunological mechanisms including antibodies production and cytokines mediated damage [2]. Sex hormones, especially estrogen and prolactin (PRL), have an important role in modulating the immune response in SLE [3].

Hyperprolactinaemia in lupus patients is associated with all types of serositis and anemia and with disease activity [4].

Pericarditis is the most common cardiac manifestations of SLE [5]. The prevalence of pericarditis and pericardial effusion documented by echocardiography varies considerably in the literature ranging from $9 \%$ up to $54 \%$ [6].

We describe a case of SLE manifesting as neuropsychiatric disease complicated by pericardial effusion in the presence of serologic abnormalities including low $\mathrm{C} 4$ and hyperprolactinaemia.

We evaluate that a patient had a good and sustained over time response to therapy with cyclophosphamide.

\section{Case Report}

A 21-year old Caucasian woman presented with malaise, intermittent fever, joint pain and stiffness.

She also developed disturbances in thought processes and an acute confusional state (delirium) associated to anxiety disorder.

She was hospitalized. Clinical examination revealed fever $\left(38.5^{\circ} \mathrm{C}\right)$, synovitis of metacarpophalengeal joints and a diffuse hair loss; she also had voice, visual and auditory hallucinations in presence of insomnia and soliloquies.

Laboratory investigations showed haemoglobin 10.4 g/l, white cell count $2.000 / \mathrm{mm}^{3}$, ESR $75 \mathrm{~mm} / \mathrm{h}, \mathrm{C} 42$ $\mathrm{mg} / \mathrm{dl}$, diffuse hypergammaglobulinaemia, PRL level 100 $\mathrm{ng} / \mathrm{ml}$ (normal $<20 \mathrm{ng} / \mathrm{ml}$ ) antinuclear antibodies present at titer 1/1280 with a diffuse pattern, anti-dsDNA, antiribosomal $\mathrm{P}$ antibody positivity. A complete hormonal panel including follicle stimulating hormone (FSH), luteinizing hormone (LH), total estrogen, progesterone, free testosterone, pregnenolone, DHEA sulphate and thyroid profile was performed and no alterations were 
found.

IgM anticardiolipin and IgM anti $\beta 2$ glycoprotein I ( $\beta 2 \mathrm{GPI})$ were negative.

To establish the CNS manifestations of SLE we performed a Nuclear Magnetic Resonance (NMR) and a Positron Emission Tomography (PET) to evaluate metabolism of the brain in course of psychiatric symptoms.

NMR did not show ischaemic and haemorragic lesions, neither hypothalamic disorders or hypophysitis.

PET did not reveal metabolism alterations in anterior parts of the brain in course of psychiatric symptoms.

The diagnosis of SLE was made and a corticosteroids pulse therapy was started.

The psychiatric consultation stated that the patient had important mood disorders and a therapy with haloperidol was also started.

After fifteen days of therapy she was afebrile and synovitis and psychoses had significantly improved. Laboratory investigations showed haemoglobin $12.0 \mathrm{~g} / \mathrm{l}$, white cell count $6.000 / \mathrm{mm}^{3}$, ESR

35 but still low C4 level and high level of prolactin. She continued on oral corticosteroid therapy.

However seven days later, just 3 weeks after initial presentation, she was readmitted with relapsing fever $\left(39^{\circ} \mathrm{C}\right)$ and chest pain of pleuritic type. The electrocardiogram showed non specific ST segment alteration, but echocardiography revealed the presence of moderate pericardial effusion (Figure 1).

Cyclophosphamide pulse therapy was then started; seven days after the patient was afebrile and subsequent echocardiographic evaluation showed a significant improvement of pericardial effusion and a normal value of prolactin.

At that time the patient had a complete disappearance of mood disease.

\section{Discussion}

The involvement of the central nervous system is one of

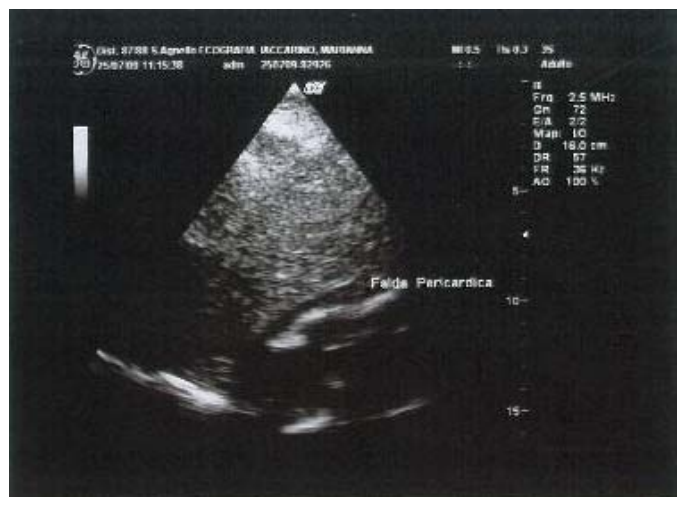

Figure 1. Echocardiography with the presence of moderate pericardial effusion. the major causes of morbidity and mortality in systemic lupus erythematosus patients and, at the same time, the less understood aspect of the disease [7]. In our case the patient presented with an acute confusional state and mood disorders requiring corticosteroids as first line therapy [8]. Of note was the inadequacy of steroid treatment in terms of clinical response that could be related to a variable neural substrate in presence of immunological disorders.

On the other hand, corticosteroids use, low C4 level anti-ds-DNA positivity are related to pericarditis, one of the commonest manifestation of SLE, and pericardial fluid may contain anti-DNA and low complement levels [4], particularly low serum C4 levels in female sex [9]. Kahl found that significantly more women had cardiac tamponade than did men and serum C4 level was lower in those with tamponade [10].

However, we assessed elevated serum levels of prolactin associated with sierositis (in this case pericarditis) and a serological activity.

In recent years, a number of reports have suggested differences in the frequency of clinical and laboratory parameters of SLE between male and female patients [11].

There was a trend that suggested that patients who have elevated level of prolactin may have an increased risk of neuropsychiatric manifestations.

The clinical picture of hyperprolactinaemia is characterized by endocrinological disorders in up to $20 \%$ of SLE patients.

However, our patient did not show alterations of endocrinological panel, neither PET and NMR revealed alterations of CNS.

In our opinion, CNS manifestations of SLE were considered as "associated" when it was an autoimmune disease originating in CNS.

Autoimmune disease determined hyperprolactinaemia and consequent psychosis? Important was the response to CYC therapy after failure of steroids and antidepressant therapy. Our patient also developed pericardial effusion combined to low C4 level persisting even on corticosteroid treatment, and she showed a complete resolution of pericardial effusion and on cyclophosphamide pulse therapy. No pericardial effusion relapse was seen on mid term follow-up. To our knowledge, this is the first case of NP-SLE complicated by pericardial effusion during steroids therapy, showing a good response on cyclophosphamide therapy both in terms of pericardial effusion resolution, neuropsychiatric manifestations resolution and normal level of prolactin [12].

\section{REFERENCES}

[1] S. G. West, "Neuropsychiatric Lupus," Rheumatic Disease Clinics of North America, Vol. 20, No. 1, 1994, pp. 
129-158.

[2] A. Afeltra, M. Vadacca, S. Galluzzo, L. Conti, A. P. Mitterhofer, G. M. Ferri, et al., "Thrombosis in Systemic Lupus Erythematosus: Congenital and Acquired Risk Factors," Arthritis \& Rheumatism, Vol. 53, No. 3, 2005, pp. 452-459. doi:10.1002/art.21172

[3] S. Shelly, M. Boaz and H. Orbach, "Prolactin and Autoimmunity," Autoimmunity Reviews, Vol. 11, No. 6-7, 2012, pp. A465-A470. doi:10.1016/j.autrev.2011.11.009

[4] M. Karimifar, A. Tahmasebi, Z. S. Bonakdar and S. Purajam, "Correlation of Serum Prolactin Levels and Disease Activity in Systematic Lupus Erythematosus," Rheumatology International, Vol. 33, No. 2, 2013, pp. 511516. doi:10.1007/S00296-011-2211-5

[5] N. E. Doherty and R. J. Siegel, "Cardiovascular Manifestations of Systemic Lupus Erythematosus," American Heart Journal, Vol. 110, No. 6, 1985, pp. 1257-1265.

[6] B. L. Man and C. C. Mok, "Serositis Related to Systemic Lupus Erythematosus: Prevalence and Outcome,” Lupus Vol. 14, No. 10, 2005, pp. 822-826.

[7] G. Sanna, M. L. Bertolaccini and M. A. Khamashta, "Neuropsychiatric Involvement in Systemic Lupus Erythematosus: Current Therapeutic Approach," Current Pharmaceutical Design, Vol. 14, No. 13, 2008, pp. 1261-
1269. doi: $10.2174 / 138161208799316401$

[8] S. D. Denburg, R. M. Carbotte and J. A. Denburg, "Corticosteroids and Neuropsychological Functioning in Patients with Systemic Lupus Erythematosus,” Arthritis \& Rheumatism, Vol. 37, No. 9, 1994, pp. 1311-1320.

[9] P. Nihoyannopoulos, P. M. Gomez, J. Joshi, S. Loizou, M. J. Walport and C. M. Oakley, "Cardiac Abnormalities in Systemic Lupus Erythematosus. Association with Raised Anticardiolipin Antibodies," Circulation, Vol. 82, No. 2, 1990, pp. 369-375.

[10] L. E. Kahl, "The Spectrum of Pericardial Tamponade in Systemic Lupus Eythematosus. Report of Ten Patients,” Arthritis \& Rheumatism, Vol. 35, No. 11, 1992, pp. 13431349.

[11] Y. Ding, J. He, J. P. Guo, Y. J. Dai, C. Li, M. Feng, R. Li and Z. G. Li, "Gender Differences Are Associated with the Clinical Features of Systemic Lupus Erythematosus," Chinese Medical Journal (English Edition), Vol. 125, No. 14, 2012, pp. 2477-2481.

[12] S. D. Denburg, R. M. Carbotte and J. A. Denburg, "Corticosteroids and Neuropsychological Functioning in Patients with Systemic Lupus Erythematosus,” Arthritis \& Rheumatism, Vol. 37, No. 9, 1994, pp. 1311-1320. 